\title{
COLOUR VISION CHANGES IN ACUTE CENTRAL SEROUS RETINOPATHY PATIENTS
}

\author{
Mustafa Saad1, Muhemmed Swadique ${ }^{2}$
}

${ }^{1}$ Resident, Department of Ophthalmology, Al Salama Eye Hospital, Perinthalmanna.

2Professor, Department of Ophthalmology, MES Medical College, Perinthalmanna.

\section{ABSTRACT}

\section{BACKGROUND}

Patients suffering from Central Serous Retinopathy (CSR) are in high risk of colour vision problems. The purpose of the study is to find the colour vision changes in patient having acute CSR with using Farnsworth D-15 Dichotomous Test.

\section{MATERIALS AND METHODS}

The study was done in 60 eyes of 30 individuals suffering from acute CSR presented in our hospital. The main objective of the study is to find out colour vision changes in acute CSR cases.

\section{CONCLUSION}

73.33\% cases the person with CSR suffers from colour vision defect.

\section{KEYWORDS}

Central Serous Retinopathy, Colour Vision, D-15.

HOW TO CITE THIS ARTICLE: Saad M, Swadique M. Colour vision changes in acute central serous retinopathy patients. J. Evolution Med. Dent. Sci. 2016;5(30):1515-1517, DOI: 10.14260/jemds/2016/357

\section{INTRODUCTION}

Central Serous Retinopathy (CSR), also known as idiopathic central serous chorioretinopathy, is a condition in which there is a collection of fluid beneath the center (the macula) of the retina. ${ }^{1}$ The retina is the nerve tissue that lines the back wall of the eye. Much like the film in a camera, the retina is sensitive to light. It transforms light energy to nerve stimuli and "sends a picture" through the optic nerve to the brain. The macula is the part of the retina that allows a person to have sharp, clear colour vision. ${ }^{2}$ Our ability to read and to see fine detail depends upon the health of the macula. The collection of fluid beneath the macula can interfere with its function. The definite cause of this condition is unknown, but the onset of symptoms may occur in a patient at a time of unusual emotional stress. CSR typically affects healthy young and middle-aged males between ages 20 and 45 years of age. ${ }^{3}$ Patients may complain of blurring of central vision, dyschromatopsia, metamorphopsia, micropsia.

Eyes with CSR had minimal relative afferent pupillary defects, reduced critical flicker-fusion thresholds, prolonged Visual Evoked Potential (VEP) latencies, increased errors on the Farnsworth-Munsell 100-hue (FM 100) test and depressed central visual fields. ${ }^{4}$ During a clinical examination, the physician can often see a well-defined, round or oval shallow elevation of the retina in the macula, which points to a diagnosis of CSR. Fundus Fluorescein angiography is performed to confirm diagnosis and assess the site of leakage. In most cases, time will be given for spontaneous resolution of the fluid before considering treatment with laser. The chance of spontaneous resolution and return of vision is excellent.

Financial or Other, Competing Interest: None.

Submission 09-03-2016, Peer Review 22-03-2016,

Acceptance 25-03-2016, Published 12-04-2016.

Corresponding Author:

Dr. Mustafa Saad,

Resident, Department of Ophthalmology,

Al Salama Eye Hospital,

Perinthalmanna.

E-mail: drsaadmustafa@gmail.com

DOI: $10.14260 /$ jemds $/ 2016 / 357$
The amount of time allowed depends upon whether this is the first occurrence of this problem and upon the size and location of the leakage. Laser treatment seals the leaking areas and help with reabsorption of the fluid. When laser treatment is used to treat CSR, a small application is usually sufficient to successfully improve or correct the problem. Visual improvement will take at least six months after the reabsorption of the fluid. However, many patients will still have a mild permanent defect, such as a loss of colour sensitivity, a small "blind spot," or some mild form of distortion. This study was conducted to find out the colour vision changes in patients with acute central serous retinopathy.

\section{MATERIALS AND METHODS}

This was a hospital based prospective study done on 60 eyes of 30 individuals (15 males and 15 females), who had visited Al Salama Eye Hospital, which is an associate institution of MES Medical College, Perinthalmanna. A complete ocular evaluation including Snellen visual acuity testing, Ishihara pseudoisochromatic colour charting, D-15 colour vision charting, slit lamp biomicroscopy, colour fundus photography (Figures $1 \& 2$ ) and optical coherence tomography (Figure 3) was done.

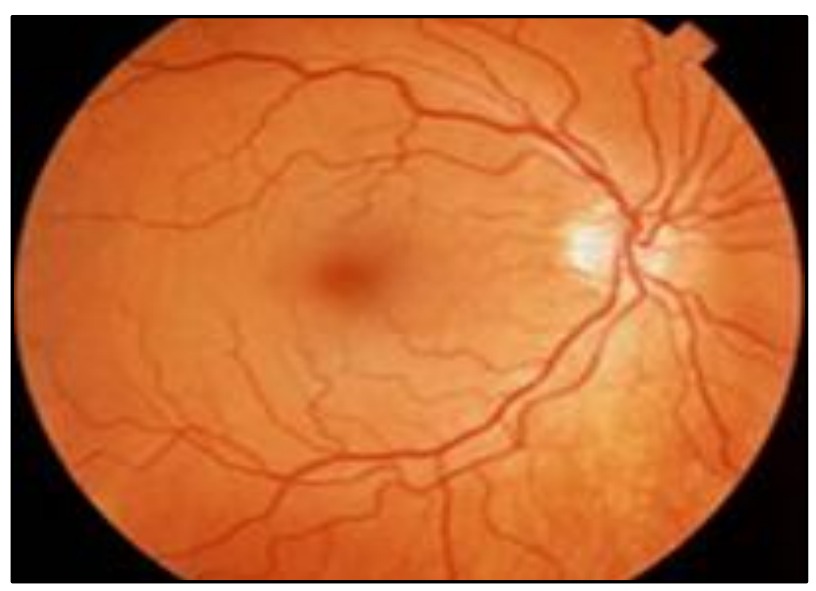

Fig. 1: Normal Fundus 


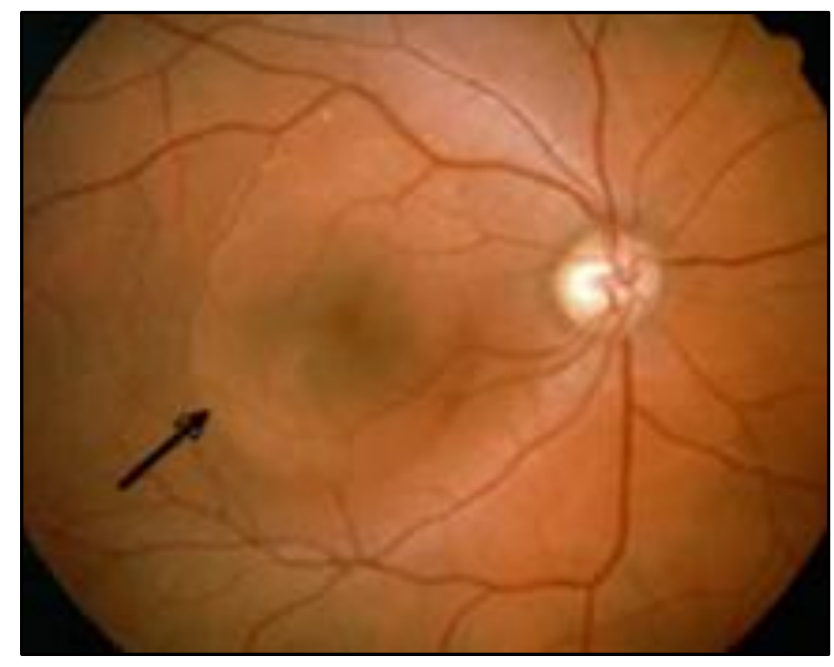

Fig. 2: CSR with Macular Fluid

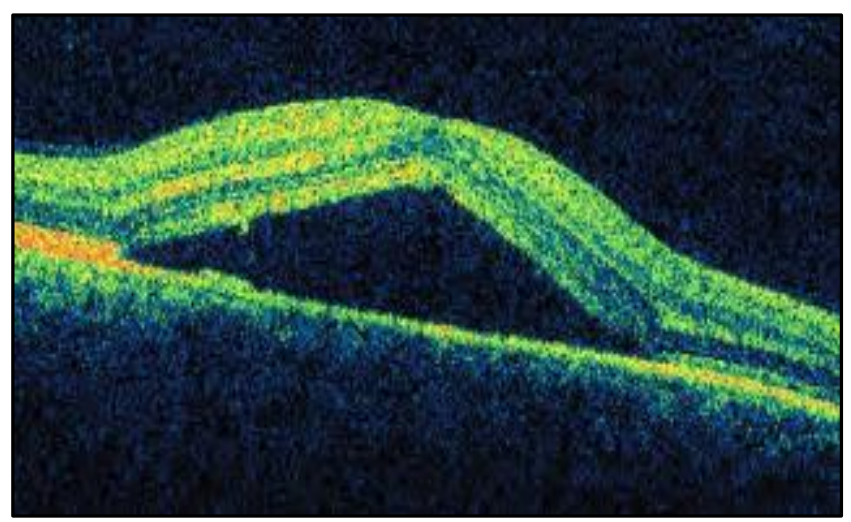

Fig. 3: OCT Picture of CSR

Patients who were newly diagnosed with acute central serous retinopathy aged between $30-80$ years and without any systemic diseases were included in this study. Patients with Uncontrolled hypertension, Diabetes and other systemic diseases who had glaucoma, cataract, who were mentally challenged were excluded from this study. Farnsworth D-15 dichotomous test is a colour blindness test, is a quick and convenient test for routine clinical use to differentiate between protan (Figure 4), deutan (Figure 5) and tritan (Figure 6) colour vision deficiencies and calculate an approximate severity of colour blindness. The disadvantage of this test is that it cannot detect some weak forms of colour vision deficiency and differentiate between dichromacies and anomalous trichromacies.

The test results are based around lines of confusions, which are associated with protan, deutan, tritan colour blindness. Vingrys and King-Smith developed in 1988, a scoring method based on colour difference vectors which make it possible to quantify the type of colour blindness by the confusion angle and severity through confusion index. The test should be performed under daylight condition. A transparent plastic box with a transparent plastic cover having 15 coloured disc, which are numbered at the back is opened and the discs are removed, then the patient undergoing the test is asked to arrange the coloured disc to create a continuum of gradual changing hues within the box, after which the cover is placed over the box and the box is put upside down and then the order of cap placement is recorded on appropriate portion of Gulden test score sheet.
Connecting the dots on circular plotting form for each test, we get a circular plot. Complete passing of the test occurs when the sequence of cap placement is exactly correct and circular plot has no cross overs. Minor error occurs when there are crossovers occurring around the circle involving 1-2 cap positions. Major error occurs when crossovers go across the circle graph often defining an axis of colour deficiency type.

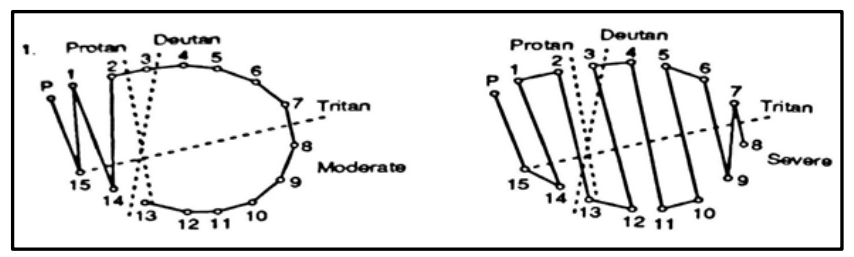

Fig. 4: Protan Defect

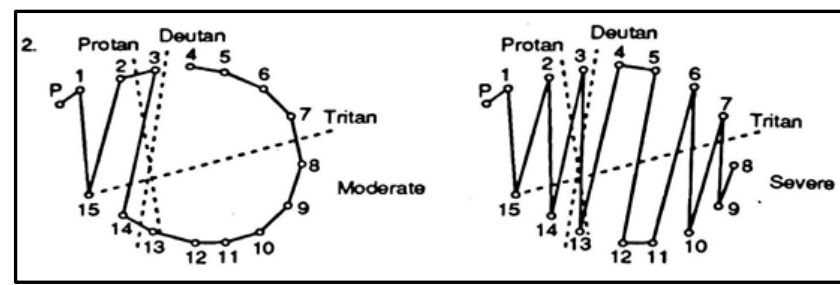

Fig. 5: Deutan Defect

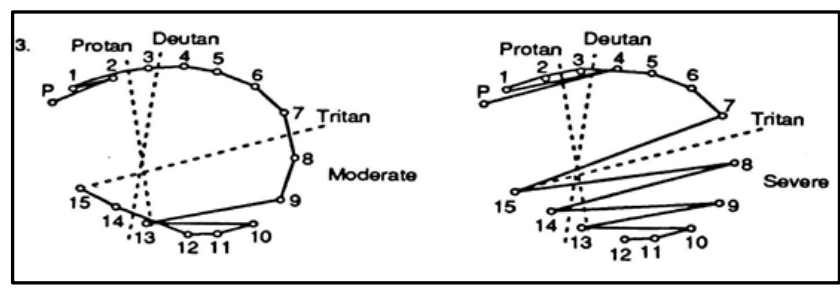

Fig. 6: Tritan Defect

\section{RESULTS}

It was observed in this study that following an attack of CSR, most of the patients had colour vision defects (Graph 1), mostly involving the blue colour. There was no correlation between the time since the active disease and the results on the colour vision tests. Farnsworth D-15 dichotomous test was found to be a useful diagnostic test and provides a viable alternative to lengthier and complicated Farnsworth Munsell 100 hue test for diagnosing colour vision defects in acute CSR patients. Most of the patients with colour vision defects are tritan defects (Graph 2).

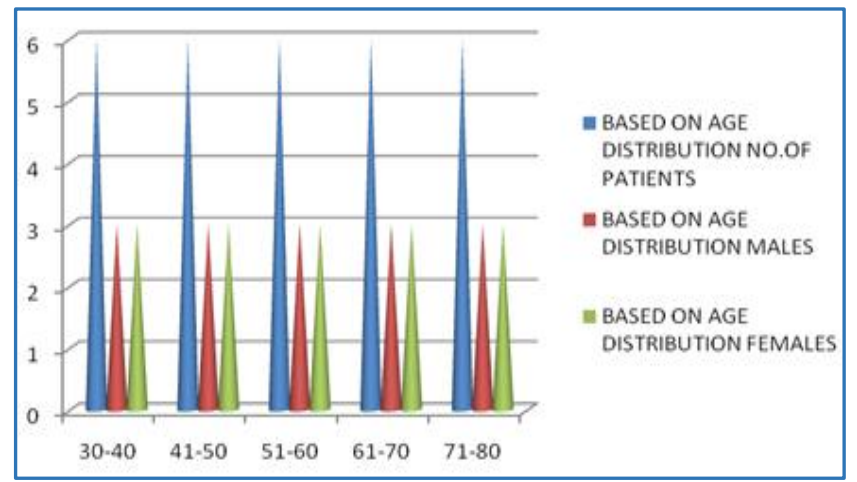

Graph 1: Based on Age Distribution 


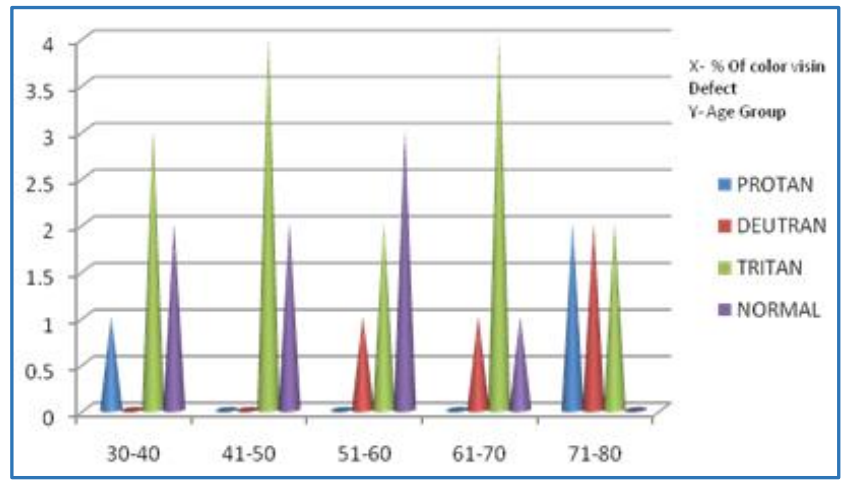

Graph 2: Shows Tritan Deficiency is more in CSR Patients

\section{DISCUSSION}

Central serous retinopathy is a disease that involves accumulation of serous fluid between photoreceptors and the retinal pigment epithelium. Its affects are two folds, one disordering of photoreceptors and two slowing of photopigment regeneration. The photo pigment alteration is thought to occur only during periods of active neurosensory detachments and serous leakage. This abnormality resolves when affected eye recovers. In a study conducted by Spitznas $M$ et al., it was observed that during periods of neurosensory detachments, cone optical densities were low and optical densities remained low following clinical resolution of the disease. 5

In a study conducted by Maaranen TH et al., it showed that of the CSR eyes 26 (67\%) had a colour vision defect, most of them in the blue area. ${ }^{6}$ In another study conducted by Dennis P. Han, James et al., showed that the most common colour defect in Optic neuritis was in the green area using
Farnsworth Munsell 100 hue tests, whereas in acute CSR patients the most common colour defect was found out to be in the blue area. ${ }^{7}$ In this study also, it was found that most of the patients suffering from acute central serous retinopathy had colour vision defect using Farnsworth D-15 dichotomous test and the most common colour deficiency found was blue.

\section{CONCLUSION}

This study concludes $73.33 \%$ of the patients had a colour vision defect and $50 \%$ of the patients having colour vision defect in acute CSR had defect in blue area.

\section{REFERENCES}

1. Taylor W. Central serous retinopathy. Seminars in Ophthalmology 1988;3(2):139-142.

2. Green C. Diseases of the macula: a practical approach. Optometry and Vision Science 2003;80(8):553-554.

3. Theodossiadis G, Tongos D. Treatment of central serous retinopathy. Ophthalmologica 1974;169(6):416-431.

4. Hasrod N, Rubin A. Colour vision: a review of the cambridge colour test and other colour testing methods. African Vision and Eye Health 2015;74(1):7.

5. Spitznas M. Pathogenesis of central serous retinopathy: a new working hypothesis. Graefe's Arch Clin Exp Ophthalmol 1986;224(4):321-324.

6. Maaranen TH, Tuppurainen KT, Mantyjarvi MI. Color vision defects after central serous chorioretinopathy. Retina 2000;20(6):633-637.

7. Coppeto JR. Central serous retinopathy, the farnsworthmunsell 100-Hue test, and prolactin. Archives of Ophthalmology 1985;103(3):323-325. 